\title{
Testing Spatial Noncommutativity via Rydberg Atoms
}

\author{
Jian-zu Zhang * \\ Institute for Theoretical Physics, Box 316, East China University of Science and \\ Technology, Shanghai 200237, P. R. China
}

\begin{abstract}
The possibility of testing spatial noncommutativity via Rydberg atoms is explored. An atomic dipole of a cold Rydberg atom is arranged in appropriate electric and magnetic field, so that the motion of the dipole is constrained to be planar and rotationally symmetric. Spatial noncommutativity leads to that the canonical angular momentum possesses fractional values. In the limit of vanishing kinetic energy, the dominate value of the lowest canonical angular momentum takes $\hbar / 2$. Furthermore, in the limit of eliminating magnetic field, the dominate value of the lowest canonical angular momentum changes from $\hbar / 2$ to $\hbar / 4$. This result is a clear signal of spatial noncommutativity. An experimental verification of this prediction is suggested.
\end{abstract}

* E-mail address: jzzhang@ecust.edu.cn 
In hinting new physics in the present round it seems that physics in noncommutative space [1] is a candidate. This is motivated by studies of low energy effective theory of D-brane with a nonzero NS-NS $B$ field background. Effects of spatial noncommutativity are only apparent near the string scale, thus we need to work at a level of noncommutative quantum field theory. But it is expected that some low energy relics of such effects may be verified by nowadays experiments, and some phenomenological low energy effects may be explored in solvable models at a level of quantum mechanics in noncommutative space(NCQM). In literature NCQM have been extensively studied [2 4].

In this paper we study the possibility of testing spatial noncommutativity via Rydberg atoms at the level of NCQM. In [5, 6] it is demonstrated that cold Rydberg atoms play an interesting role of realizing analogs of Chern-Simons theory [7, 8]. An atomic dipole of a cold Rydberg atom is arranged in appropriate electric and magnetic field, so that the motion of the dipole is constrained to be planar and rotationally symmetric. In this case the Röntgen term of the Hamiltonian takes on a form of a Chern-Simons interaction. This term makes interesting contribution to dynamics. Furthermore, in an appropriate optical trapping field the elimination of dipole's kinetic energy could be achieved physically, and the canonical angular momentum spectrum changes from integers to positive half integers. An experimental verification of the Chern-Simons feature of the angular momentum is allowed.

In noncommutative space new features appear. Spatial noncommutativity leads to that the canonical angular momentum spectrum possesses fractional values [9]. In the limit of vanishing kinetic energy the dominate value of the lowest canonical angular momentum takes $\hbar / 2$. Spatial noncommutativity permits a further limiting process of eliminating magnetic field that the dominate value of the lowest canonical angular momentum changes from $\hbar / 2$ to $\hbar / 4$. This result is a clear signal of spatial noncommutativity. A possibility of testing spatial noncommutativity via cold Rydberg atoms is suggested.

NCQM Algebra. In order to develop the NCQM formulation we need to specify the phase space and the Hilbert space on which operators act. The Hilbert space can consistently be taken to be exactly the same as the Hilbert space of the corresponding commutative system [2]. 
As for the phase space we consider both space-space noncommutativity (space-time noncommutativity is not considered) and momentum-momentum noncommutativity. There are different types of noncommutative theories, for example, see a review paper [10].

In the case of simultaneously space-space noncommutativity and momentum-momentum noncommutativity the consistent NCQM algebra [9] are:

$$
\left[\hat{x}_{i}, \hat{x}_{j}\right]=i \xi^{2} \theta \epsilon_{i j}, \quad\left[\hat{x}_{i}, \hat{p}_{j}\right]=i \hbar \delta_{i j}, \quad\left[\hat{p}_{i}, \hat{p}_{j}\right]=i \xi^{2} \eta \epsilon_{i j},(i, j=1,2)
$$

where $\theta$ and $\eta$ are the constant parameters, independent of position and momentum; $\epsilon_{i j}$ is an antisymmetric unit tensor, $\epsilon_{12}=-\epsilon_{21}=1, \epsilon_{11}=\epsilon_{22}=0$; The scaling factor $\xi=$ $\left(1+\theta \eta / 4 \hbar^{2}\right)^{-1 / 2}$.

The Hamiltonian of a Rydberg atom in electric and magnetic field [5, 6] is (summation convention is used):

$$
\hat{H}=\frac{1}{2 \mu}\left(\hat{p}_{i}+\frac{1}{2} g \epsilon_{i j} \hat{x}_{j}\right)^{2}+\frac{1}{2} \kappa \hat{x}_{i}^{2}=\frac{1}{2 \mu} \hat{p}_{i}^{2}+\frac{1}{2 \mu} g \epsilon_{i j} \hat{p}_{i} \hat{x}_{j}+\frac{1}{2} \mu \omega^{2} \hat{x}_{i}^{2},
$$

where the co-ordinates $\hat{x}_{i}$ refer to the laboratory frame of the Rydberg atom. ${ }^{1}$ The parameter $\mu$ is the mass of the atom. The electric field $\vec{E}$ acts radially in the $x-y$ plane, $E_{i}=-\mathcal{E} \hat{x}_{i},(i=1,2)$, where $\mathcal{E}$ is a constant, $(\mathrm{i}=1,2)$, and the constant magnetic field $\vec{B}$ aligns the $z$ axis. The constant parameters $g=2 q B$ and $\kappa=2 q \mathcal{E}, q(>0)$ is dipole's electric charge. ${ }^{2}$ The term $g \epsilon_{i j} \hat{p}_{i} \hat{x}_{j} /(2 \mu)$ takes the Chern-Simons interaction. The frequency $\omega=\left[g^{2} /\left(4 \mu^{2}\right)+\kappa / \mu\right]^{1 / 2}$, where the dispersive "mass" term $g /(2 \mu)$ comes from the presence of the Chern-Simons term.

The motivation of considering both space-space and momentum-momentum noncommutativity is as follows. There are different ways to construct creation-annihilation operators. We first construct the deformed annihilation-creation operators $\left(\hat{a}_{i}, \hat{a}_{i}^{\dagger}\right)(i=1,2)$ at the non-perturbation level which are related to the variables $\left(\hat{x}_{i}, \hat{p}_{i}\right)$ :

$$
\hat{a}_{i}=\sqrt{\frac{\mu \omega}{2 \hbar}}\left(\hat{x}_{i}+\frac{i}{\mu \omega} \hat{p}_{i}\right) .
$$

\footnotetext{
1 The Rydberg atom is treated as a structureless dipole moment. In reality it has the internal atomic structure. For the following discussions effects of the internal structure are extremely small, and hence can be forgotten.

2 Rydberg atoms are sensitive to external electric fields. Even Relatively modest electric fields may ionize Rydberg atoms. If we ignore the Stark shift of a Rydberg state of effective principal quantum number $n^{*}$ we obtain the classical electric field $E_{c}$ for ionization $E_{c}=\left(16 n^{* 4}\right)^{-1}$ a.u. $=3.2 \cdot\left(n^{*}\right)^{-4} \cdot 10^{8} \mathrm{~V} / \mathrm{cm}$. In our discussion the electric field $E_{i}$ should satisfy the condition $|E|<E_{c}$.
} 
(3) and the NCQM algebra (11) show that the operators $\hat{a}_{i}^{\dagger}$ and $\hat{a}_{j}^{\dagger}$ for the case $i \neq j$ do not commute. When the state vector space of identical bosons is constructed by generalizing one-particle quantum mechanics, because of such a noncommutativity the operators $\hat{a}_{1}^{\dagger} \hat{a}_{2}^{\dagger}$ and $\hat{a}_{2}^{\dagger} \hat{a}_{1}^{\dagger}$ applied successively to the vacuum state $|0\rangle$ do not produce the same physical state. In order to maintain Bose-Einstein statistics at the non-perturbation level described by $\hat{a}_{i}^{\dagger}$ the basic assumption is that operators $\hat{a}_{i}^{\dagger}$ and $\hat{a}_{j}^{\dagger}$ should be commuting. This requirement leads to a consistency condition of NCQM algebra

$$
\eta=\mu^{2} \omega^{2} \theta
$$

From (11), (3) and (41) it follows that the commutation relations of $\hat{a}_{i}$ and $\hat{a}_{j}^{\dagger}$ read

$$
\left[\hat{a}_{1}, \hat{a}_{1}^{\dagger}\right]=\left[\hat{a}_{2}, \hat{a}_{2}^{\dagger}\right]=1,\left[\hat{a}_{1}, \hat{a}_{2}\right]=0 ; \quad\left[\hat{a}_{1}, \hat{a}_{2}^{\dagger}\right]=i \xi^{2} \mu \omega \theta / \hbar
$$

The first three equations in (5) are the same commutation relations as the one in commutative space.

The last equation in (5) codes effects of spatial noncommutativity. We emphasize that it is consistent with all principles of quantum mechanics and Bose-Einstein statistics.

If momentum-momentum is commuting, $\eta=0$, we could not obtain the third equation in (5). It is clear that in order to maintain Bose-Einstein statistics for identical bosons at the level of $\hat{a}_{i}$ and $\hat{a}_{i}^{\dagger}$ we should consider both space-space noncommutativity and momentummomentum noncommutativity.

Now we consider perturbation expansions of $\left(\hat{x}_{i}, \hat{p}_{j}\right)$ and $\left(\hat{a}_{i}, \hat{a}_{j}^{\dagger}\right)$. The NCQM algebra (11) has different perturbation realizations [4]. We consider the following consistent ansatz of the perturbation expansions of $\hat{x}_{i}$ and $\hat{p}_{i}$

$$
\hat{x}_{i}=\xi\left[x_{i}-\theta \epsilon_{i j} p_{j} /(2 \hbar)\right], \quad \hat{p}_{i}=\xi\left[p_{i}+\eta \epsilon_{i j} x_{j} /(2 \hbar)\right]
$$

where $\left[x_{i}, x_{j}\right]=\left[p_{i}, p_{j}\right]=0,\left[x_{i}, p_{j}\right]=i \hbar \delta_{i j}$. In commutative space the relations between the variables $\left(x_{i}, p_{i}\right)$ and the annihilation-creation operators $\left(a_{i}, a_{i}^{\dagger}\right)$ are $a_{i}=\sqrt{\mu \omega /(2 \hbar)}\left[x_{i}+\right.$ $\left.i p_{i} /(\mu \omega)\right]$, where $\left[a_{i}, a_{j}\right]=\left[a_{i}^{\dagger}, a_{j}^{\dagger}\right]=0,\left[a_{i}, a_{j}^{\dagger}\right]=\delta_{i j}$. Inserting these relations into (6) , using (44) and comparing the results with (3), we obtain the perturbation expansions of $\hat{a}_{i}$ and $\hat{a}_{i}^{\dagger}$

$$
\hat{a}_{i}=\xi\left[a_{i}+i \mu \omega \theta \epsilon_{i j} a_{j} /(2 \hbar)\right], \quad \hat{a}_{i}^{\dagger}=\xi\left[a_{i}^{\dagger}-i \mu \omega \theta \epsilon_{i j} a_{j}^{\dagger} /(2 \hbar)\right]
$$


(11) and (3)-(71) are consistent each other.

Spectrum of Rydberg Atoms. As in commutative space the angular momentum is defined as an exterior product $\hat{J}=\epsilon_{i j} \hat{x}_{i} \hat{p}_{j}$. From (11) and (4) it follows that $[\hat{J}, \hat{H}]=0$. Thus $\hat{H}, \hat{J}$ constitute a complete set of observables of the system.

In the following our attention is focused on the perturbation investigation of $\hat{H}$ and $\hat{J}$. Using (6]) we obtain

$$
\hat{H}=\frac{1}{2 M}\left(p_{i}+\frac{1}{2} G \epsilon_{i j} x_{j}\right)^{2}+\frac{1}{2} K x_{i}^{2}=\frac{1}{2 M} p_{i}^{2}+\frac{1}{2 M} G \epsilon_{i j} p_{i} x_{j}+\frac{1}{2} M \Omega^{2} x_{i}^{2},
$$

where the effective parameters $M, G, K$ and $\Omega$ are defined as $1 /(2 M) \equiv \xi^{2}\left[c_{1}^{2} /(2 \mu)+\kappa \bar{\theta}^{2} / 2\right]$, $G /(2 M) \equiv \xi^{2}\left(c_{1} c_{2} / \mu+\kappa \bar{\theta}\right), M \Omega^{2} / 2 \equiv \xi^{2}\left[c_{2}^{2} /(2 \mu)+\kappa / 2\right], K \equiv M \Omega^{2}-G^{2} /(4 M)$, and $c_{1}=1+g \bar{\theta} / 2, c_{2}=g / 2+\bar{\eta}, \bar{\theta}=\theta /(2 \hbar), \bar{\eta}=\eta /(2 \hbar)$.

(8) is exactly solvable [5, 6]. We introduce new variables $\left(X_{\alpha}, P_{\alpha}\right), X_{a}=\sqrt{M \Omega /\left(2 \omega_{a}\right)} x_{1}-$ $\sqrt{1 /\left(2 M \Omega \omega_{a}\right)} p_{2}, X_{b}=\sqrt{M \Omega /\left(2 \omega_{b}\right)} x_{1}+\sqrt{1 /\left(2 M \Omega \omega_{b}\right)} p_{2}, P_{a}=\sqrt{\omega_{a} /(2 M \Omega)} p_{1}+\sqrt{M \Omega \omega_{a} / 2} x_{2}$, $P_{b}=\sqrt{\omega_{b} /(2 M \Omega)} p_{1}-\sqrt{M \Omega \omega_{b} / 2} x_{2}$, where $\omega_{a}=\Omega+G /(2 M), \quad \omega_{b}=\Omega-G /(2 M)$, and define new annihilation operators $A_{\alpha}=\sqrt{\omega_{\alpha} /(2 \hbar)} X_{\alpha}+i \sqrt{\hbar /\left(2 \omega_{\alpha}\right)} P_{\alpha},(\alpha=a, b)$. Then the Hamiltonian (8) decomposes into two uncoupled harmonic oscillators of unit mass and frequencies $\omega_{a}$ and $\omega_{b}$ :

$$
\hat{H}=H_{a}+H_{b}, \quad H_{\alpha}=\hbar \omega_{\alpha}\left(A_{\alpha}^{\dagger} A_{\alpha}+1 / 2\right), \quad(\alpha=a, b)
$$

By a similar procedure we obtain the perturbation expansion of $\hat{J}$

$$
\begin{aligned}
\hat{J} & =\epsilon_{i j} x_{i} p_{j}-\xi^{2}\left(\bar{\theta} p_{i} p_{i}+\bar{\eta} x_{i} x_{i}\right) \\
& =\hbar\left(A_{b}^{\dagger} A_{b}-A_{a}^{\dagger} A_{a}\right)-\left(A_{a}^{\dagger} A_{a}+A_{b}^{\dagger} A_{b}+1\right) \mathcal{J}_{0}, \quad \mathcal{J}_{0}=\xi^{2} \mu \omega \theta \hbar
\end{aligned}
$$

where the zero-point angular momentum $\mathcal{J}_{0}=\langle 0|J| 0\rangle$ codes effects of spatial noncommutativity. It worth noting that it takes fractional value. The consistency condition (4) of NCQM algebra is crucial in the derivation of (10). The second line of (10) is derived by using a relation $M \Omega=\mu \omega$ which is obtained from (44).

Dynamics in the limit of Vanishing Kinetic Energy. In the limit of vanishing kinetic energy, $E_{k} \rightarrow 0$, the Hamiltonian (8) shows non-trivial dynamics. In this limit there are constraints which should be carefully considered. For this purpose it is more convenient 
to work in the Lagrangian formulism. First we identify the limit of vanishing kinetic energy in the Hamiltonian with the limit of the mass $M \rightarrow 0$ in the Lagrangian. In (8) in the limit of vanishing kinetic energy, $\frac{1}{2 M}\left(p_{i}+\frac{1}{2} G \epsilon_{i j} x_{j}\right)^{2}=\frac{1}{2} M \dot{x}_{i} \dot{x_{i}} \rightarrow 0$, the Hamiltonian $H$ reduces to $H_{0}=\frac{1}{2} K x_{i} x_{i}$. The Lagrangian corresponding to the Hamiltonian (8) is $L=\frac{1}{2} M \dot{x}_{i} \dot{x}_{i}+\frac{1}{2} G \epsilon_{i j} x_{i} \dot{x_{j}}-\frac{1}{2} K x_{i} x_{i}$. In the limit of $M \rightarrow 0$ this Lagrangian reduces to $L_{0}=\frac{1}{2} G \epsilon_{i j} x_{i} \dot{x_{j}}-\frac{1}{2} K x_{i} x_{i}$. From $L_{0}$ the corresponding canonical momentum is $p_{0 i}=$ $\partial L_{0} / \partial \dot{x}_{i}=\frac{1}{2} G \epsilon_{j i} x_{j}$, and the corresponding Hamiltonian is $H_{0}^{\prime}=p_{0 i} \dot{x}_{i}-L_{0}=\frac{1}{2} K x_{i} x_{i}=H_{0}$. Thus we identify the two limiting processes. We emphasize when the potential is velocity dependent the limit of vanishing kinetic energy in the Hamiltonian does not corresponds to the limit of vanishing velocity in the Lagrangian. If the velocity approached zero in the Lagrangian there would be no dynamics. The Hamiltonian (2) and its massless limit have been studied by Dunne, Jackiw and Trugenberger [8].

The first equation of (8) shows that in the limit $E_{k} \rightarrow 0$ there are constraints ${ }^{3}$

$$
C_{i}=p_{i}+\frac{1}{2} G \epsilon_{i j} x_{j}=0 .
$$

The Poisson brackets of constraints (11) are $\left\{C_{i}, C_{j}\right\}_{P}=G \epsilon_{i j} \neq 0$, so that the corresponding Dirac brackets of canonical variables $x_{i}, p_{j}$ can be determined [12], $\left\{x_{1}, p_{1}\right\}_{D}=$ $\left\{x_{2}, p_{2}\right\}_{D}=1 / 2,\left\{x_{1}, x_{2}\right\}_{D}=-1 / G,\left\{p_{1}, p_{2}\right\}_{D}=-G / 4$. The Dirac brackets of $C_{i}$ with any variables $x_{i}$ and $p_{j}$ are zero that the constraints (11) are strong conditions and can be used to eliminate the dependent variables. If we select $x_{1}$ and $p_{1}$ as the independent variables, from (111) we obtain $x_{2}=-2 p_{1} / G, p_{2}=G x_{1} / 2$. The above Dirac brackets show that the corresponding quantization condition of the independent variables $x_{1}$ and $p_{1}$ is $\left[x_{1}, p_{1}\right]=i \hbar / 2$. In order to rewrite $H_{0}$ in the traditional form we introduce new variables $q=\sqrt{2} x_{1}$ and $p=\sqrt{2} p_{1}$, which satisfy the normal quantization condition $[q, p]=i \hbar$. We introduce the effective mass $\mu^{*} \equiv G^{2} / 2 K$ and effective frequency $\omega^{*} \equiv K / G$, and rewrite the Hamiltonian $H_{0}$ as $H_{0}=\frac{1}{2 \mu^{*}} p^{2}+\frac{1}{2} \mu^{*} \omega^{* 2} q^{2}$. Then we define a new annihilation operator $A=\sqrt{\mu^{*} \omega^{*} / 2 \hbar} q+i \sqrt{\hbar / 2 \mu^{*} \omega^{*}} p$, and rewrite the Hamiltonian $H_{0}$ as $H_{0}=\hbar \omega^{*}\left(A^{\dagger} A+1 / 2\right)$. Similarly, we rewrite the angular momentum $\hat{J}$ in (10) as $J_{0}=\hbar \mathcal{J}_{0}^{*}\left(A^{\dagger} A+1 / 2\right)$, where $\mathcal{J}_{0}^{*}=1-\xi^{2}(G \bar{\theta} / 2 \hbar+2 \bar{\eta} / G \hbar)$. The eigenvalues of $H_{0}$ and

\footnotetext{
${ }^{3}$ In this example the symplectic method [11] leads to the same results as the Dirac method for constrained quantization, and the representation of the symplectic method is much streamlined.
} 
$J_{0}$ are, respectively, $E_{n}^{*}=\hbar \omega^{*}(n+1 / 2), \mathcal{J}_{n}^{*}=\hbar \mathcal{J}_{0}^{*}(n+1 / 2),(n=1,2, \cdots)$. In the limit case $E_{k} \rightarrow 0$ the corresponding lowest angular momentum is $\hbar \mathcal{J}_{0}^{*} / 2$ whose dominate value is $\hbar / 2$.

Because of spatial noncommutativity a further limiting process of diminishing magnetic field also leads to non-trivial dynamics. In this limit the parameter $g \rightarrow 0$, the frequency $\omega \rightarrow \omega_{0} \equiv \sqrt{\kappa / \mu}$, the consistency condition (44) is rewritten as $\bar{\eta}=\mu^{2} \omega_{0}^{2} \bar{\theta}$, and $\xi \rightarrow \xi_{0}=\left(1+\mu^{2} \omega_{0}^{2} \bar{\theta}^{2}\right)^{-1 / 2}$. The effective parameters $M, \Omega, G$ and $K$ reduce, respectively, to the following effective parameters $\tilde{M}, \tilde{\Omega}, \tilde{G}$ and $\tilde{K}$, which are defined by $\tilde{M} \equiv$ $\left[\xi_{0}^{2}\left(1 / \mu+\kappa \bar{\theta}^{2}\right)\right]^{-1}=\mu, \tilde{\Omega}^{2} \equiv \xi_{0}^{2}\left(\kappa / \mu+\bar{\eta}^{2} / \mu^{2}\right)=\omega_{0}^{2}, \tilde{G} \equiv 2 \xi_{0}^{2}(\mu \kappa \bar{\theta}+\bar{\eta})=2 \xi_{0}^{2} \mu \kappa \theta / \hbar$ and $\tilde{K} \equiv \tilde{M} \tilde{\Omega}^{2}-\tilde{G}^{2} /(4 \tilde{M})=\kappa\left(1-\xi_{0}^{4} \mu \kappa \theta^{2} / \hbar^{2}\right)$. Thus in this limit $H_{0}$ and $J_{0}$ reduce, respectively, to the following $\tilde{H}_{0}$ and $\tilde{J}_{0}$ :

$$
\begin{aligned}
\tilde{H}_{0} & =\hbar \tilde{\omega}\left(\tilde{A}^{\dagger} \tilde{A}+1 / 2\right), \\
\tilde{J}_{0} & =\hbar \tilde{\mathcal{J}}_{0}\left(\tilde{A}^{\dagger} \tilde{A}+1 / 2\right), \quad \tilde{\mathcal{J}}_{0}=1-\xi_{0}^{2}(\tilde{G} \bar{\theta} / 2+2 \bar{\eta} / \tilde{G})=\left(1-\xi_{0}^{4} \mu \kappa \theta^{2} / \hbar^{2}\right) / 2,
\end{aligned}
$$

where the annihilation operator $\tilde{A}=\sqrt{\tilde{\mu} \tilde{\omega} / 2 \hbar} q+i \sqrt{\hbar / 2 \tilde{\mu} \tilde{\omega}} p$, the effective mass $\tilde{\mu} \equiv$ $\tilde{G}^{2} /(2 \tilde{K})$ and frequency $\tilde{\omega} \equiv \tilde{K} / \tilde{G}$. From (12) we conclude that the dominate value of the lowest angular momentum $\hbar \tilde{\mathcal{J}}_{0} / 2$ is $\hbar / 4$. Unlike the term $2 \xi^{2} \bar{\eta} /(G \hbar) \sim \mu \kappa \theta /\left(g \hbar^{2}\right) \sim 0$ in $\mathcal{J}_{0}^{*}$, here the term $2 \xi_{0}^{2} \bar{\eta} / \tilde{G}=1 / 2$ in $\tilde{\mathcal{J}}_{0}$. This leads to the difference between the dominate values of $\mathcal{J}_{0}^{*}$ and $\tilde{\mathcal{J}}_{0}$. If we define the angular momentum with scalar terms $\bar{\eta} \hat{x}_{i} \hat{x}_{i}+\bar{\theta} \hat{p}_{i} \hat{p}_{i}$ as in [4], we obtain the same conclusion. This dominate value $\hbar / 4$ of the lowest angular momentum explores the essential new feature of spatial noncommutativity. ${ }^{4}$

Testing Spatial Noncommutativity via Rydberg Atoms. Following [5, 6, we arrange a cold Rydberg atom in the electric and magnetic fields with the above suggested arrangement. Assume that the atomic dipole confined in a plan is prepared in its energy ground state and interacts with a laser beam of a Laguerre-Gaussian form. The expectation value of the angular momentum in the long time limit [14] shows two distinct resonances at $\omega_{a}, \omega_{b}$. In an appropriate laser trapping field the speed of the atom can be slowed

\footnotetext{
${ }^{4}$ In the limit of vanishing magnetic field the Hamiltonian of this system reduces to the Hamiltonian of a harmonic oscillator. In commutative space the dynamics of a harmonic oscillator in the limit of vanishing kinetic energy does not possesses similar constraints. As the kinetic energy decreased, the potential energy decreases, so that the oscillation gets weeker and weeker. Thus in commutative space in both limits of vanishing kinetic energy and magnetic field there is no dynamics.
} 
to the extent that the kinetic energy term in (8) may be removed [16]. As the kinetic energy diminished, only one resonance remains at $\omega^{*}=K / G$, and the dominate value of the corresponding lowest angular momentum is $\hbar / 2$. Furthermore, as the magnetic field eliminated, the parameter $g$ approaches zero, the resonance occurs at $\tilde{\omega}=\tilde{K} / \tilde{G} \sim \hbar /(\mu \theta)$, and the dominate value of the corresponding lowest angular momentum shifts to $\hbar / 4$. Since a Laguerre-Gaussian beam carries orbital angular momentum along its direction of propagation [13, an atom moving in such a beam is subject to a radiation-induced torque, which is proportional to the eigenvalue of mode's orbital angular momentum [15]. This suggests that a Laguerre-Gaussian beam supplies a suitable probe for the above angular momentum resonances.

Of course, any attempt to detect effects of spatial noncommutativity, if any, is a challenging enterprise. In the above case the dominate value of the lowest angular momentum $\hbar / 4$ is independent of the parameter $\theta$, but the frequency $\tilde{\omega}$ is $\theta$ dependent. There are different bounds on the parameter $\theta$ set by experiments. The space-space noncommutative theory from string theory violates Lorentz symmetry and therefore strong bounds can be placed on the parameter $\theta$, the existing experiments [17] give $\theta /(\hbar c)^{2} \leq(10 T e V)^{-2}$. Comparing with the above estimation, other bounds on $\theta$ exist: measurements of the Lamb shift [2] give a weaker bound; clock-comparison experiments [18] claim a stronger bound. The magnitude of $\theta$ is surely extremely small; the frequency $\tilde{\omega}$ is surely extremely large. ${ }^{5}$

This work has been partly supported by the National Natural Science Foundation of China under the grant number 10074014 and by the Shanghai Education Development Foundation.

\footnotetext{
${ }^{5}$ The dominate value of the frequency $\tilde{\omega}$ is $\tilde{\omega}=\tilde{K} / \tilde{G} \approx \hbar /(2 \mu \theta)$. If we take $\mu c^{2}=2 G e V$ and $\theta /(\hbar c)^{2} \leq\left(10^{4} \mathrm{GeV}\right)^{-2}$ we obtain $\tilde{\omega} \geq 10^{32} \mathrm{~Hz}$.
} 


\section{References}

[1] A. Connes, M. R. Douglas, A. Schwarz, JHEP 9802, 003 (1998) hep-th/9711162 M. R. Douglas, C. M. Hull, JHEP 9802, 008 (1998) hep-th/9711165 F. Ardalan, H. Arfaei, M. M. SheikhJabbari, JHEP 9902, 016 (1999) hep-th/9810072 S-C. Chu, P-M. Ho, Nucl. Phys. B550, 151 (1999) hep-th/9812219 S-C. Chu, P-M. Ho, Nucl. Phys. B568, 447 (2000) hep-th/9906192 V. Schomerus, JHEP 9906, 030 (1999) hep-th/9903205 N. Seiberg and E. Witten, JHEP 9909:032 (1999) hep-th/9908142

[2] M. Chaichian, M. M. Sheikh-Jabbari, A. Tureanu, Phys. Rev. Lett. 86, 2716 (2001) hep-th/0010175.

[3] J. Gamboa, M. Loewe, J. C. Rojas, Phys. Rev. D64, 067901 (2001) hep-th/0010220 A. Hatzinikitas, I. Smyrnakis, J. Math. Phys. 43113 (2002) hep-th/0103074 A. Smailagic, E. Spallucci, Phys. Rev. D65, 107701 (2002) hep-th/0108216 P-M. Ho, H-C. Kao, Phys. Rev. Lett. 88, 151602 (2002) hep-th/0110191.

[4] V. P. Nair, A. P. Polychronakos, Phys. Lett. B505, 267 (2001) hep-th/2011172.

[5] C. Baxter, Phys. Rev. Lett. 74, 514 (1995).

[6] Jian-zu Zhang, Phys. Rev. Lett. 77, 44 (1996).

[7] S. Deser, R. Jackiw, S. Templeton, Ann. Phys. (N. Y.) 140, 372 (1982); S. C. Zhang, T. H. Hansson, S. Kivelson, Phys. Rev. Lett. 62, 82 (1989); G. V. Dunne, R. Jackiw, C. A. Trugenberger, Phys. Rev. D41, 661 (1990).

[8] G. V. Dunne, R. Jackiw, C. A. Trugenberger, Phys. Rev. D41, 661 (1990).

[9] Jian-zu Zhang, Phys. Lett. B584, 204 (2004).

[10] M. R. Douglas, N. A. Nekrasov, Rev. Mod. Phys. 73, 977 (2001) hep-th/0106048

[11] L. Faddeev, R. Jackiw, Phys. Rev. Lett. 60, 1692 (1988). 
[12] M. J. W. Müller-Kirsten, Jian-zu Zhang, Phys. Lett. A202, 241 (1995).

[13] L. Allen, M. W. Beijersbergen, R. J. C. Spreeuw, J. P. Woerdman, Phys. Rev. A45, 8185 (1992).

[14] R. Loudon, The Quantum Theory of Light (Clarendon, Oxford, 1983), 2nd. ed.

[15] M. Babiker, W. L. Power, L. Allen, Phys. Rev. Lett. 73, 1239 (1994).

[16] F. Shimizu, K. Shimizu, H. Takuma, Opt. Lett. 16, 339 (1991).

[17] S. M. Carroll, J. A. Harvey, V. A. Kostelecky, C. D. Lan, T. Okamoto, Phys. Rev. Lett. 87, 141601 (2001) hep-th/0105082.

[18] I. Mocioiu, M. Pospelov, R. Roiban, Phys. Lett. B489, 390 (2000) hep-th/00005191. 